\title{
Enhanced homestead food production
}

Glob. Food Sec. 24, 100335 (2020)

Enhanced homestead food production (EHFP) programmes address structural determinants of malnutrition through the promotion of nutrient-sensitive agriculture. Such programmes have gained traction among policymakers and practitioners as a strategy for improving food security and nutrition, as the developmental approaches also promote health, longerterm productivity and income sustainability. However, it is still unclear under what conditions EHFP can deliver all these benefits or what is their added value when combined with other interventions such as micronutrient supplementation or industrial food fortification.

Nick Dragojlovic, from the University of British Columbia, and colleagues explored this question by conducting an economic evaluation of Family Farms for the Future (FF4F) - an EHFP programme implemented in rural Cambodia, where high poverty rates and lack of agricultural infrastructure pose serious threats to food security. The programme was evaluated on the basis of costs of delivery, impact on food production (with incremental changes calculated over ten years) and impacts on health outcomes (measured in disabilityadjusted life years (DALYs) averted as a result of improved nutrient intake).

The study shows that FF4F is associated with a net societal benefit when sustained for ten years, including an increase in the value of agricultural production (US $\$ 1$, 602 per household) and an estimated 44.6 DALYs averted due to zinc and vitamin A deficiency per 1,000 households. According to literature cited by the authors, FF4F's resulting benefit-cost ratio (BCR) of $0.43-$ 6.11 overlaps with the BCR range of zinc and vitamin A supplementation programmes in least developed countries (1.22-9.4), but is below the BCR of fortification or biofortification interventions (10-30). Thus, even though EHFP seems less cost-effective than biofortification regarding zinc and vitamin A, it may have an equal or even better cost-benefit ratio in the long run.

The limitations acknowledged by the authors - common to impact evaluations of such programmes - relate to the acquisition of temporally consistent data that, accounting for the effect of climate fluctuations, can more accurately attribute health outcomes to interventions. Nevertheless, this study provides a more nuanced picture of EHFP and elicits the importance of specific factors when picking a strategy for nutrition security; for instance, EHFP may be most suited to populations that are hard to reach with fortification interventions, or that may particularly benefit from the additional welfare benefits of EHFP (for example, improved food security and household income). Finally, the FF4F case study also draws attention to the importance of education, capacity building, and political and institutional conditions for the continuity of the programme until its benefits can be reaped.

Juliana Gil

Published online: 13 January 2020 https://doi.org/10.1038/s43016-019-0024-y 